\title{
Prism adaptation to dynamic events
}

\author{
DOUGLAS P. FIELD \\ University of North Texas, Denton, Texas \\ THOMAS F. SHIPLEY \\ Temple University, Philadelphia, Pennsylvania \\ and \\ DOUGLAS W. CUNNINGHAM \\ Logicon Technical Services, Dayton, Ohio
}

\begin{abstract}
In the present study, we explored adaptation to prism-displaced dynamic and static events under conditions of minimal information. Many of our interactions with the world are dynamic and involve reaching for or intercepting moving objects. The consequences (or feedback) of those interactions entail the presence or absence of physical contact with the moving objects. In this study, humans learned, with only haptic feedback, to intercept optically displaced falling balls. To eliminate visual feedback, the falling balls disappeared behind an occluder (which systematically varied in size across groups) prior to either striking or missing a subject's hand. As occluder size decreased, adaptation increased. With minimum occluder sizes, the greatest adaptation occurred around the training position, and adaptation decreased as distance between training and testing positions increased. The results can best be described in terms of a generalization gradient centered around the training position. This generalization gradient was not present when subjects were trained with ecologically similar static arrays. Implications for models of adaptation are discussed.
\end{abstract}

Humans adapt to changes in the relation between where things are seen and where they are felt. Such changes occur gradually during maturation (e.g., when the length of arms and legs increase) and abruptly when one must act in a new medium with a new refractive index (e.g., when one begins wearing eyeglasses or reaches for something below the surface of a liquid). Nonhumans can also behave effectively in such circumstances. For example, the archer fish swims smoothly through water and can knock insects into the water by aiming and shooting a stream of water from below the surface-clearly, it can deal effectively with the refractory properties of both water and air. While it is possible that such behavior requires specialized innate neural mechanisms, the behavior of animals that deal with multiple media, such as archer fish and wading birds, may also develop as a result of general learning processes that serve to adapt spatially oriented behavior to a variety of contexts (Goldstein \& Hall, 1990).

Studies of this type of learning in humans have typically employed prisms that laterally offset a visual field containing static elements. Adaptation to visual displace-

This research was supported by a Temple University Research Fellowship to D.P.F. and NSF Research Grant BNS 93-96309 to T.F.S. The research was conducted while all three authors were at Temple University. The authors thank Ronald Baenninger, Philip Hineline, Gordon Redding, and an anonymous reviewer for their thoughtful suggestions, Robert Kirk for his help with data collection and analysis, and J. D. Brown for his help in constructing the experimental chamber. Correspondence should be addressed to T. F. Shipley, Department of Psychology, Temple University, Philadelphia, PA 19122. ment has been a fertile ground for empirical investigations and conceptual interpretations for over 100 years (for reviews, see Bedford, 1993b; Harris, 1965, 1980; Redding \& Wallace, 1997; Welch, 1978). When subjects are first exposed to visual displacement, their behavior with respect to what is seen (e.g., pointing at, reaching for, or touching) is notably ineffective. With continued interaction with the visually displaced environment, performance improves and becomes more effective (i.e., subjects become increasingly accurate in their reaching and pointing). When the prisms are removed, a decrement in accuracy is seen (errors are now made in the direction opposite to the prior visual displacement). This difference or change in pointing is often used as a measure of adaptation. ${ }^{1}$

The focus on static arrays, where adaptation occurs with respect to stationary targets, has led to neglect of an important aspect of our interactions with the world-its dynamic nature. Often, the objects we reach for, or move to intercept, are themselves in motion. Although prism adaptation has been shown to occur in the presence of dynamic stimulation (e.g., adaptation while pointing at moving targets, Wallace, 1975; while walking around, Held, 1961, and Mikaelian, 1970; and while throwing balls, Redding \& Wallace, 1995), we know little of whether a general learning process can account for adaptation in both static and dynamic contexts. In the one study (Wallace, 1975) that provided a direct comparison of adaptation to moving versus stationary targets, slight differences in adaptation between these two contexts were found; however, the exact nature of such differences has yet to be fully assessed. In the present paper, we examine prism 
adaptation to dynamic events and to static events and explore differences in adaptation and the generalization of adaptation in these two cases.

Static and dynamic environments differ in at least two important ways: (1) Dynamic environments offer optical information for action that is not present in a static environment, and (2) many of the actions made with respect to dynamic environments differ qualitatively from actions to a static environment. Although research on prism adaptation has yet to explore these differences, there is a developing literature within the area of event perception that is relevant to the focus of the present investigaiion. A number of researchers have examined the information provided by the motion of an object or surface that can guide precise action. Most of this research has focused on interactions with moving objects: perceiving upcoming contacts with them (e.g., Kaiser \& Phatak, 1993; Kaiser \& Proffitt, 1984; Kim, Turvey, \& Carello, 1993; Tresilian, 1991) or intercepting and catching them (e.g., Lee \& Reddish, 1981; McBeath, 1990; McBeath, Shaffer, \& Kaiser, 1995; Michaels \& Oudejans, 1992). In each of these cases, the perceptual experiences and actions of the subjects required an analysis of the dynamic aspects of the scene; effective action could not have occurred if the static properties of a scene were the only basis for action.

Actions that occur in dynamic and static contexts must differ in character. Peper, Bootsma, Mestre, and Bakker (1994, p. 591) noted that to "be successful in interceptive actions, like catching or hitting a ball, the movement behavior of the actor has to be very precisely attuned to the spatiotemporal characteristics of the event." This highlights three important differences between reaching for or pointing at stationary objects and intercepting dynamic objects. First, in intercepting or catching, the hand does not move to the location of the target (as it does with stationary targets); the hand moves to a location that is spatially and temporally separated from the visual location of the target. The second difference is temporal in nature: In order to effectively intercept, the hand must occupy tiee interception point at a particular time and must arrive there prior to that time. These qualitative differences in action, and in information, inherent to interacting with moving objects may result in different patterns of learning in the two cases. Finally, the outcome of reaching for or intercepting moving objects is often physical contact with the object. As a result, feedback about the success or failure of an action is largely haptic in nature. Research on prism adaptation has typically involved visual feedback during pointing tasks (i.e., the hand is seen to have aligned, or failed to align, with its intended target). Some studies have employed haptic (often referred to as tactile) feedback (e.g., Howard, Craske, \& Templeton, 1965; Lackner, 1974; Welch, 1978); however, no clear consensus on its efficacy has been reached by these researchers. In addition to providing a direct comparison of adaptation to static and dynamic events, the present study also allowed an examination of the efficacy of haptic feedback.

An effective strategy for exploring the nature, extent, and persistence of adaptation, as well as perceptual learn- ing in general, is to arrange for adaptation to occur under conditions of minimal information (Bedford, 1989; Shepard, 1984). Information for adaptation is minimized by limiting the extent or mode of target exposure and the range or number of spatial locations used during adaptation. The effects of prism experience are then assessed by either simply testing for the occurrence of adaptation (e.g., Held \& Hein, 1958; Moulden, 1971) or comparing adaptation at trained and untrained locations (e.g., Baily, 1972; Bedford, 1989, 1993a; Cohen, 1966).

To study adaptation to optical displacement of dynamic events, we have adapted Bedford's $(1989,1993$ a) general preparation for investigating how experience with prism displacement at a single location will affect performance across a range of locations (both trained and nontrained). In Bedford's original experiments, her subjects pointed at light-emitting diodes (LEDs) illuminated on a black background. She limited the amount of information available during training by running her subjects in the dark and providing visual feedback for pointing responses by illuminating an LED, affixed to their index finger, only when they were pointing directly at a given target (in all but one of Bedford's experiments, subjects were not informed of the optical displacement present during adaptation). She found that, after her subjects were trained to point at only one location while wearing prisms, a relatively uniform shift in pointing without prisms was observed across a broad range of locations on the horizontal arc in front of her subjects. When her subjects were trained at two locations (at which the visual offset was either in the same or opposing directions) and then tested, the resulting changes in pointing across all locations approximated a linear function and were best described as a linear interpolation across all locations that passed through the two trained locations. Bedford argued that these results suggest that the system is constrained in how it can react to change and that perceptual learning in general reflects the system's attempt to align two dimensions (e.g., visual and proprioceptive space).

In the present study, we adapted these general procedures in order to investigate how training to intercept visually displaced falling objects at one location generalizes to performance at untrained locations. To examine adaptation under minimal information conditions in a manner analogous to Bedford's, it was necessary to constrain the subject's interactions such that, during training, (1) antecedent target exposures were limited to a single location on a horizontal plane through which a falling object would pass and (2) the feedback that occurred at that point was limited to only hapric information.

\section{EXPERIMENT 1 Adaptation to Dynamic Events}

In order to provide exposure to visual displacement at only one location in the horizontal plane, it is necessary to hide the subjects' view of their hand. Bedford did this by running subjects in the dark and illuminating an LED on her subject's index finger only when it was pointing 
directly at the target. In Experiment 1, we achieved this by obscuring the last portion of the falling object's path and thereby completely eliminating sight of the hand and arm, but allowing feedback to occur in a naturalistic manner: Accurate pointing resulted in physical contact with the falling targets, and inaccurate responses were indicated by a lack of contact.

Because the view of the hand (and also the falling object at the contact point) was occluded, there was a temporal and spatial separation between the antecedent visual information and the haptic consequence. In addition to examining the generalization of prism adaptation to novel locations, the extent of the spatiotemporal separation between antecedent dynamic visual stimuli and consequent haptic feedback was parametrically varied and also examined in Experiment 1.

Method
Subjects
Sixty-eight Temple University undergraduates participated in
partial fulfillment of an introductory psychology course's require-
ments. All subjects had normal or corrected-to-normal vision. Three
of the subjects were left-handed. Because there were no differences
in the pattern of results when the data were analyzed including and
excluding the left-handed subjects, their data are included in the
analyses.
Data were discarded from 8 of the subjects who participated in
this experiment. Six were discarded due to failure to meet the train-
ing criteria (described in the Procedure section) within 120 trials,
and 2 were discarded on the basis of postsession questioning-these
subjects reported a difference between the goggles used during
training and those used during testing.

\section{Apparatus}

The experimental chamber (a schematic illustration is shown in Figure 1), which measured $244 \times 94 \times 176 \mathrm{~cm}$, was painted flat black. The chamber enclosed the subjects on three sides and top, but was open at the rear. The subjects were seated in an adjustableheight chair in front of a chinrest positioned $109 \mathrm{~cm}$ above the floor and affixed to a shelf in the front of the chamber. There were two openings (one above and one below the shelf) in the front of the chamber. The lower opening $(12 \times 94 \mathrm{~cm})$, positioned at approximately knee height (its bottom was $57 \mathrm{~cm}$ above the floor), allowed the subject to position the hand below a falling ball without the hand or arm being visible. The upper opening allowed the subject to see the ball before it was dropped and a portion of its path as it fell. Multicolored, solid rubber balls $(2.6 \mathrm{~cm}$ in diameter) were used. Each ball was dropped from a wooden bar that was mounted $36 \mathrm{~cm}$ above and $43.5 \mathrm{~cm}$ in front of the chinrest $(82 \mathrm{~cm}$ above the center of the opening for the hand). Balls were dropped from 1 of 13 horizontal positions that were centered around the vertical position of the subject's right eye and were arranged such that, on average, they were $4^{\circ}$ apart. The distance between each position was varied slightly to avoid a regular array that would be symmetrical across the subject's line of sight. These 13 positions were marked so as to be visible only to the experimenter (although the subject could see the ball when it was held up to the position indicator). Horizontal variations in positions ranged from $21.3^{\circ}$ to the left of a point directly in front of the subject's right eye to $28.4^{\circ}$ to the right of that point (here, we adopt the convention of indicating left and right with negative and positive values, respectively). The elevation of the drop positions above the subject's right eye ranged from $36^{\circ}$ to $40^{\circ}$. The actual positions measured from the point directly in front of subject's right eye were $-17,-13.6,-9.8,-6.8,-3.4,0,2.6,5.1,8.2,12.4,16.2,19$, and $23.5 \mathrm{~cm}$. The position used for training was $+8.2 \mathrm{~cm}\left(+10.7^{\circ}\right)$.

When it was necessary to keep the falling ball from touching the subject's hand, a tray cushioned with a nylon screen was placed in the area between the hand and viewing apertures. When it was in

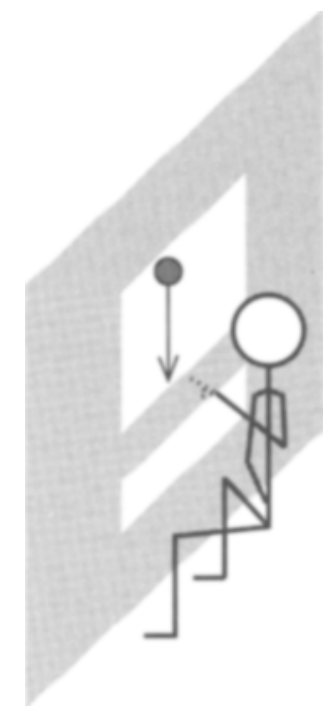

Minimum Occlusion

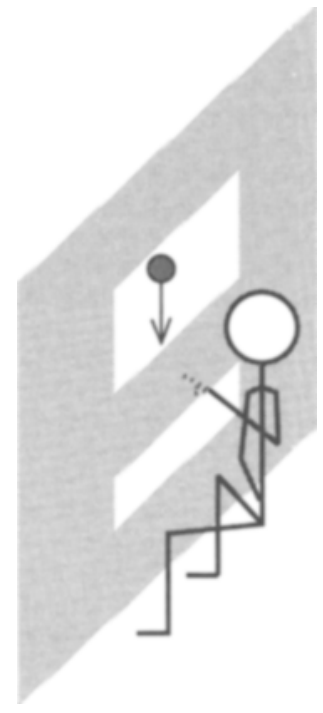

Intermediate Occlusion

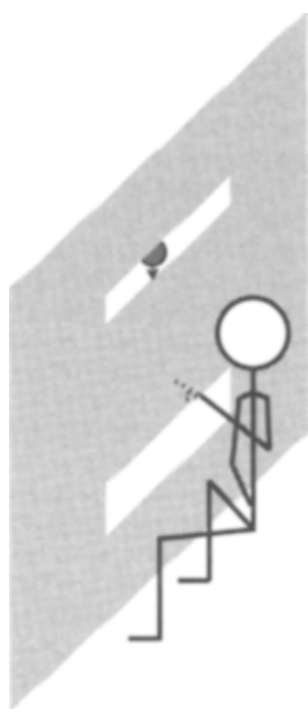

Maximum Occlusion

Figure 1. Schematic of the experimental chamber showing the three viewing aperture conditions. The subjects saw the falling balls through the top opening and attempted to touch them by positioning their hand appropriately through the lower opening. 
place, the subject could not see this tray. However, the subject's hand position could still be seen by the experimenter, so that its position could be recorded. With this tray in place, the subject could see the balls drop as usual, but haptic feedback was prevented.

Illumination during all sessions was provided by two 4-W bulbs mounted above the drop bar and out of the subject's view. A floor fan was placed just outside the experimental chamber to attenuate potential auditory location information.

Viewing aperture. To vary the length of the path seen by the subject, the size of the viewing aperture was adjusted by raising its lower edge. Three aperture sizes were used. When the aperture was at its largest, the subject could see the first $58.5 \mathrm{~cm}$ of the balls' 82 $\mathrm{cm}$ path (to the center of the hand opening). This was achieved by setting the lower edge of the aperture to $24 \mathrm{~cm}$ above the top of the hand opening. We refer to this as the minimum condition as it is the smallest amount of occlusion which is sufficient to block the subject's view of their hand. Shrinking the viewing aperture, by raising the height of the lower edge to 34.5 and $63.5 \mathrm{~cm}$ above the top of the hand slot (leaving 46 and $11.5 \mathrm{~cm}$ of the ball's path visible), composed the intermediate and maximum conditions, respectively. After a ball was dropped, it was visible for $345 \mathrm{msec}$ in the minimum condition, $306 \mathrm{msec}$ in the intermediate condition, and $153 \mathrm{msec}$ in the maximum condition. A total of $409 \mathrm{msec}$ was required for the ball to fall from the drop bar to the center of the hand opening.

Response recording. A Panasonic Super VHS video camera was mounted directly over the ball-dropping area to record finger positions on each trial. To ensure the visibility of the subject's fingers under the dim lighting conditions, a strip of white masking tape ( $2.5 \mathrm{~cm}$ wide) was wrapped around each subject's index finger, between the first and second joints. Following the test sessions, one of two observers scored each subject's test trials by replaying the trials in slow motion and recording the lateral error in finger position at the time the ball contacted the net. The subjects typically responded by pointing and holding a position, so there was little change in position during the last $200 \mathrm{msec}$ of each trial. Reliability measures were provided by having the two observers independently code 5 subjects. Interobserver reliability was very high $(r=.998)$.

Prisms. All subjects wore welder's goggles that restricted viewing to the right eye throughout the experiment. Two pairs of goggles were used. In one, the standard goggles, a flat $4.5 \times 3.3 \mathrm{~cm}$ piece of Plexiglas was mounted over the right eye opening. In the other, the prism goggles, a base-left 20 -diopter prism $\left(11.58^{\circ}\right.$ offset to the right) was placed in the right eye opening. In order to limit the discriminability of prism goggles and standard goggles due to chromatic aberrations caused by the prism, monochromatic (green) gels were mounted in the right lens of both pairs of goggles.

\section{Procedure}

To allow comparisons between groups trained with and without prisms (prism and standard) at each of the three occluder heights (minimum, intermediate, and maximum), a $2 \times 3$ design was utilized. Each group was exposed to only one occluder height. These six groups are designated by the level of occlusion and the goggles used during training (e.g., minimum-prism, intermediate-prism, minimum-standard, etc.).

Instructions. The subjects were brought into the room containing the experimental chamber and seated outside of the chamber while a set of instructions was read out loud. The instructions noted that the experiment was about hand-eye coordination under conditions with limited vision and without view of one's hands or arms. The subject was shown the balls and the chamber. Before the subject was positioned in the chamber, each of the movements required in the experiment was modeled by the experimenter and practiced by the subject. The subject was then seated in the chamber, and the room lights were turned off. With the subject's eyes closed and chin in the rest, the training phase goggles (prism for the prism groups, and standard for the standard groups) were fitted by the experimenter. The subject was asked to attempt to place his/her hand so that a small rubber ball, released from the drop bar, would contact the tip of the right index finger. The subject could not see any part of the hands or arms during the sessions. Each session included a training phase (20-30 $\mathrm{min})$ and a test phase $(5 \mathrm{~min})$, both taking place under dim light. In both phases, the subject remained seated in the chamber. Depending on which group a subject had been randomly assigned to, the apparatus was equipped with a minimum, intermediate, or maximum occlusion aperture.

Training phase. In the training phase, balls were dropped from a single position $8.2 \mathrm{~cm}\left(+10.7^{\circ}\right)$ to the right of the vertical position above the subject's right eye. Each subject was exposed to the following sequence of steps in the training trials: (1) The subject's hand was to be held open, with fingers spread and palm up. (2) Hand was to be held open, fingers together, palm up. (3) Hand closed, palm up, with only the index finger extended. (4) After four sequential trials on which the ball contacted the index finger, the main training phase began, during which the subject moved his/her right hand from the left knee such that the falling ball touched the index finger.

Progression through the training steps was determined by evolving accuracy criteria. In each of these steps, the subject moved the right hand from the left knee, through the hand opening to a point below the ball. The experimenter maintained a constant response rate by verbally cuing in a steady cadence. The cuing sequence repeated was "knee" (hand was placed on knee), "point" (experimenter positioned the ball, and the subject moved the hand under the ball), "drop" (experimenter dropped the ball). The ball was held in position for each trial between 1 and $1.5 \mathrm{sec}$ before dropping. For Steps $1-3$, the completion criterion was four in a row correct (i.e., ball must contact the hand). Once Step 4 began, training continued for a minimum of 60 trials and until the subject met a completion criterion of 8 of 16 trials on which the ball hit the index finger. Forty-seven subjects had met this criterion at the end of 60 trials, and 15 other subjects met the criterion in under 120 trials.

Test phase. Following the training phase, a testing phase was implemented using the same procedures with the following exceptions: The goggles used during training (standard or prism) were removed and the standard goggles were placed on the subject. The subject was instructed to keep his/her eyes closed and move his/her head around to "get any kinks out of your neck." The subject was told the goggles were being taken off to keep them from fogging up. Each subject was exposed to three series of 13 test trials, for a total of 39 trials. In each series, balls were dropped from each of the 13 positions along the drop bar. The order of drop positions in each series was randomly assigned. To prevent haptic feedback during the testing phase, the balls landed in a cushioned tray placed above the subject's hand. The subject was told the tray had been installed and was instructed to place the hand under where the ball should drop even though he/she would not be able to feel it. Following the session, the room was returned to normal light levels, and the subject was required to remain seated in the room for $5 \mathrm{~min}$. While the subject was seated, he/she was asked three questions by the experimenter: (1) "What was the experiment about-what were we investigating?" (2) "Did you have any difficulty getting your hand or finger under the balls?" (3) "Did you notice anything different about the goggles in the beginning and end of the session?"

\section{Results and Discussion}

The results of Experiment 1 are presented in Figures 2-5. In Figure 2, the mean pointing error at each location is given for the six groups. The horizontal axes represent the spatial positions of the 13 test locations. The vertical axes represent the mean pointing error-the horizontal 

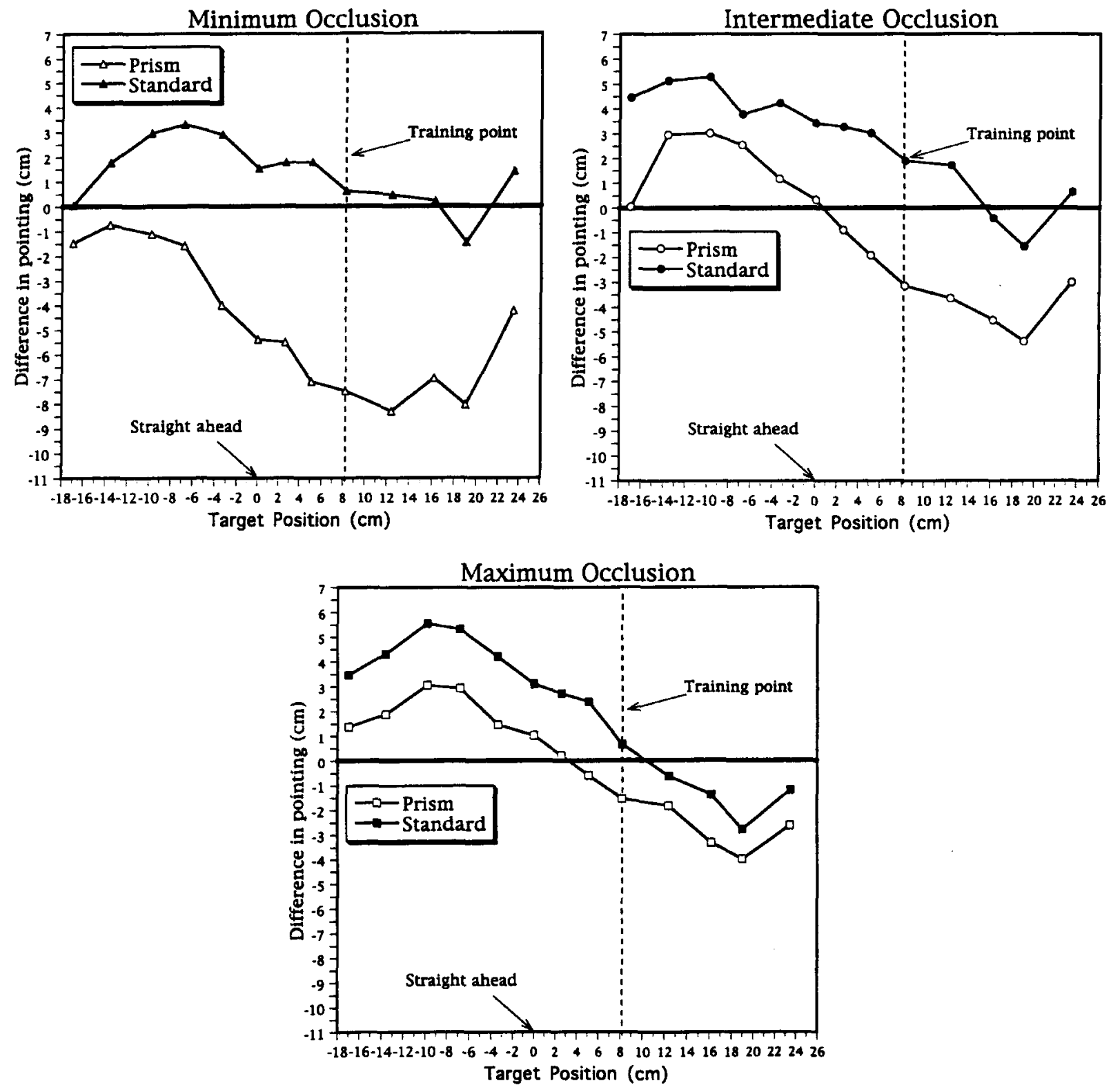

Figure 2. Pointing errors following training at one location with or without prism goggles for the three occluder heights used in Experiment 1 . Differences between the ball location and mean pointing location are plotted as a function of ball-dropping position for the prism and standard conditions for each occluder height. The dashed vertical line indicates the training location; the thick black horizontal line represents no pointing error.

distance between the point directly under the falling ball and the mean position of the subjects' index fingers. The thick solid black line indicates zero error. Group means were calculated by averaging all test trials for all subjects at each position. The standard deviations of pointing errors for all positions and groups are presented in Figure 3.

The effects of prism experience can best be assessed by examining the differences in test trial performance, at each position, between the groups trained with prism goggles and those trained with standard goggles. To this end, difference scores were calculated by subtracting the standard group means from the prism group means at each of the 13 positions for each of the occluder heights. These difference scores are presented in Figure 4.

Two aspects of Figure 4 are important. The first is that the extent of adaptation increased as the size of the occluder decreased; greater adaptation was found with the larger viewing apertures. Second, with decreasing occluder heights, the greatest adaptation occurred around the training position, and a generalization gradient cen- 

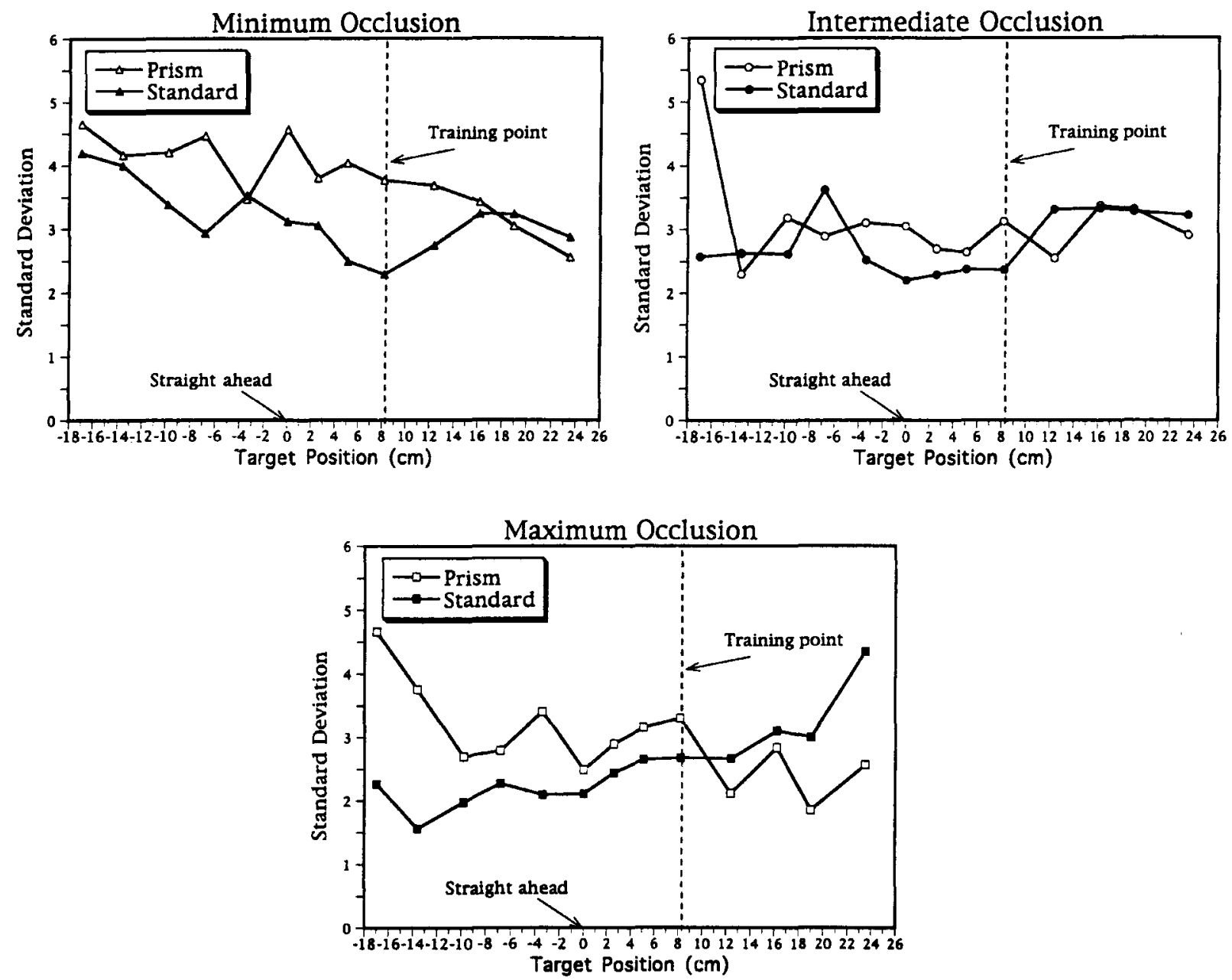

Figure 3. Standard deviations of pointing errors within each group in Experiment 1. The standard deviations in error are plotted as a function of ball-dropping position for the prism and standard conditions for each occluder height.

tered around this position was evident. Progressively less adaptation was seen as the distance between training and testing positions increased.

To analyze the pattern of adaptation, the subjects' pointing errors were subjected to a four-way analysis of variance (ANOVA), with type of goggles (prism and standard) and occluder height (maximum, intermediate, and minimum) as between-subjects factors, and test series (three levels) and target position (13 levels) as withinsubjects factors. All four main effects were significant. The significant difference between the prism and standard groups indicates that adaptation occurred in this experiment $[F(1,54)=41.1, p<.0001]$.

The main effects of occluder height and position $[F(2,54)=6.6, p<.03$, and $F(2,108)=16.9, p<.0001$, respectively] confirm that increases in adaptation occurred with decreases in occluder height and that the degree of pointing error varied as a function of target position. The main effect of test series $[F(12,648)=49.6, p<.0001]$ suggests that the amount of adaptation decreased over re- peated test trials. The decrease in pointing error occurred only in the prism groups, as reflected in the significant interaction between goggles and test series $[F(2,108)=$ $4.0, p<.03$ ]. With continued pointing, even in the absence of feedback, the average error across all positions decreased from -3.18 to $-1.29 \mathrm{~cm}$. Other researchers have found similar decrements in adaptation (i.e., decay or readaptation) under procedures ranging from the simple passage of time to the inclusion of either visual or haptic feedback (e.g., Beckett \& Melamed, 1980; Melamed, Moore, \& Beckett, 1979).

Three other two-way interactions were significant. The occluder height $\times$ goggles interaction $[F(2,54)=$ $3.3, p<.05]$ indicates that there was an effect of occluder height only for the prism groups $[F(2,54)=9, p<.001$, and $F<1$, for prism and standard groups, respectively]. Similarly, the interaction between goggles and position $[F(12,648)=2.869, p<.0008]$ reflects the difference in adaptation between the prism and standard groups. The occluder height $\times$ position interaction $[F(24,648)=$ 


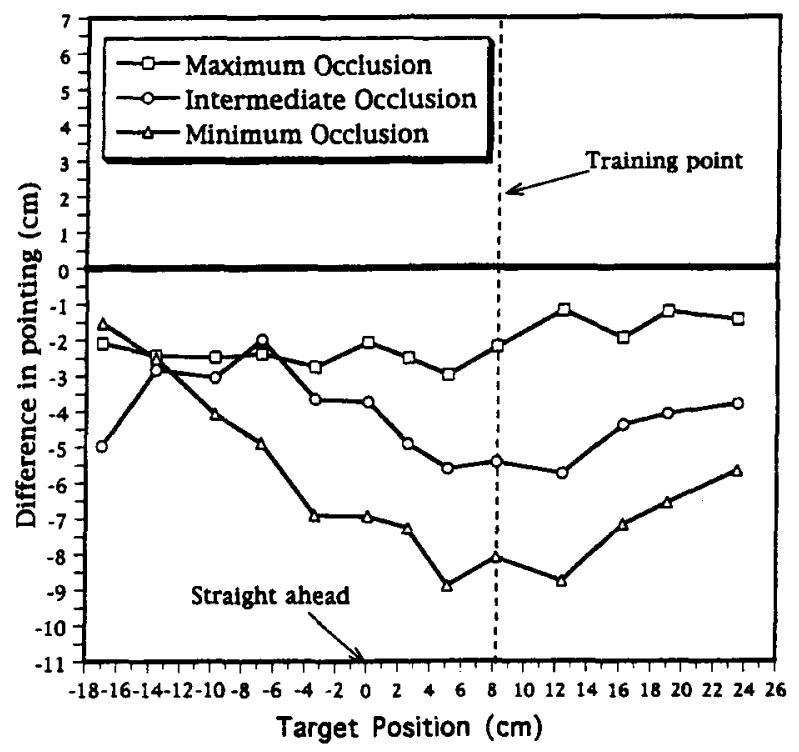

Figure 4. Pointing differences between the standard and prism groups in Experiment 1. The mean pointing difference between the standard and prism groups is plotted as a function of balldropping position for the three occluder heights.

$1.794, p<.02]$ indicates that the pattern of variation across positions changed as a function of occluder height (see Figure 4). Neither the occluder $\times$ test series nor the position $\times$ test series interactions were significant $[F<1$, and $F(24,1,296)=1.337, p>.12$, respectively].

Only one of the four three-way interactions was significant: the occluder height $\times$ goggles $\times$ position interaction $[F(24,648)=1.736, p<.02]$. This most likely reflects a pattern of changes in pointing where changes were evident only around the training position in the prism groups, and the degree of change varied as a function of occluder height. The remaining three-way interactions and the four-way interaction were nonsignificant (all $F \mathrm{~s}<1.5$, all $p \mathrm{~s}>.17$ ).

To further examine the structure of the variations in pointing error across target positions and to determine the function that best describes these results, trend analyses were performed. As a measure of adaptation, the mean pointing location of the subjects in the standard group was subtracted from all of the individual pointing scores in the prism groups. Two-way ANOVAs (test series and position) were performed on these difference scores for each occluder height. There was no main effect of position in the maximum occluder group's ANOVA, so trend analyses were performed only on the minimum and intermediate occluder groups. The first six orders (i.e., linear, quadratic, cubic, etc.) were examined. In the minimum occlusion group, the first- and second-order components were significant (i.e., there were linear and quadratic trends to the data) $[F \mathrm{~s}(1,108)>47, p \mathrm{~s}<.0001]$. The higher order components were not significant (all $F_{\mathrm{s}}<1.15, p \mathrm{~s}>.28$ ). For the intermediate occluder group, the first-, third-, and fourth-order components were significant [all $F \mathrm{~s}(1,108)>5.3, p \mathrm{~s}<.03]$. The second-, fifth-, and sixth-order components were not significant $(F \mathrm{~s}<$ $1.5, p \mathrm{~s}>.27$ ).

Since the trend analysis indicates some degree of nonlinearity in both the intermediate group and the minimum group, we fit a Gaussian function and, for comparison, a linear function to each data set. Figure 5 shows the best-fitting Gaussian and linear functions. The $r^{2} \mathrm{~s}$ for the Gaussian functions were $.96, .64$, and .62 for the minimum, intermediate, and maximum occlusions, re-
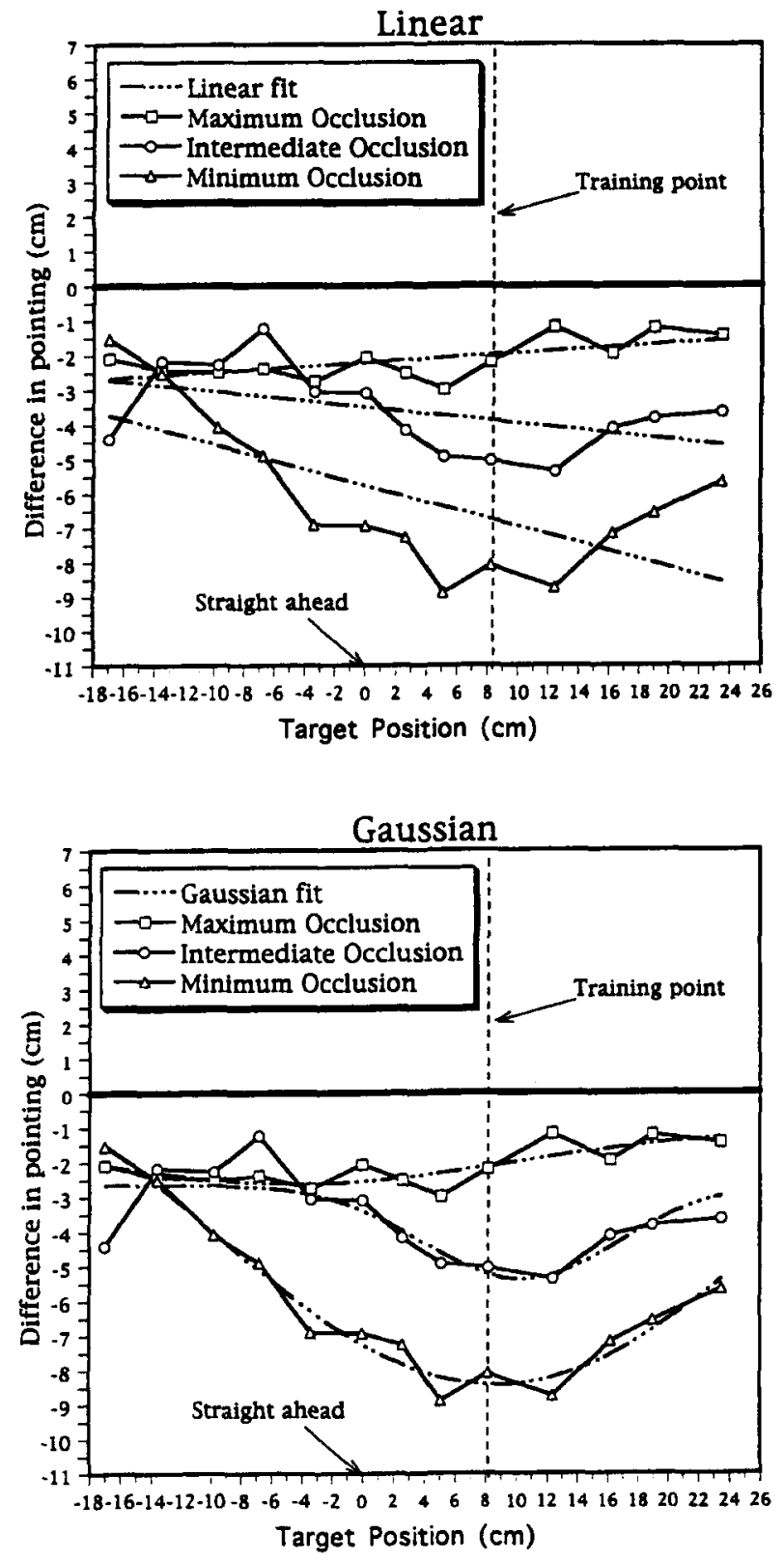

Figure 5. The best-fitting linear and Gaussian curves for the pointing differences between the prism and standard groups for each of the three occluder heights used in Experiment 1. 
spectively. As suggested by visual inspection of the curves, the linear function did not fit as well as the Gaussian $\left(r^{2} \mathrm{~s}=.45, .23\right.$, and .38 , for the minimum, intermediate, and maximum groups, respectively). When Bedford (1989, Experiment 1) examined the relation between training at a particular location and testing at nontrained locations, a global shift in pointing across the full range of untrained locations was observed. The linear function reported by Bedford differs markedly from the results of our Experiment 1.

\section{EXPERIMENT 2 Adaptation to Static Events}

The differences between the findings of Experiment 1 and Bedford's may reflect fundamental differences in how we learn to interact with static and dynamic environments. Alternatively, these differences might have arisen due to some other difference between our procedures and hers. One possibility is that the dynamic displays used in Experiment 1 were merely more complex than Bedford's. Bedford's targets appeared along a single dimension as a stationary point on a horizontal arc in front of the subjects, thus providing little if any visual differences between stimuli other than location. In contrast, intercepting movements necessarily require target displays with multiple dimensions. Although the point of contact between hand and ball in Experiment 1 was unidimensional, the dynamic visual targets were multidimensional: Each ball was dropped from a point along one dimension and traveled a path perpendicular to that dimension. The visible trajectory (i.e., the path) of the falling balls might have specified the precise lateral position of the target stimulus to a greater degree than a simple stationary LED (like those used by Bedford) and, thus, might account for the differences in degree of adaptation and the occurrence of a spatial generalization gradient.

Explicitly comparing adaptation to static and dynamic contexts required a situation, similar to the one used in Experiment 1 , containing formally similar stimulus structures and actions, yet not providing any visual change over time. A stationary multidimensional array provided by a rod, in place of the falling balls, contains all of the extended structure and feedback of the dynamic displays. ${ }^{2}$ If the higher dimensionality, or any other procedural difference, rather than the dynamic nature of the targets accounts for the generalization gradients seen in Experiment 1 , then such multidimensional static arrays should produce similar gradated adaptation functions.

In Experiment 2, using the minimal information procedure employed in Experiment 1, we explicitly contrasted dynamic to static stimuli and examined the extent to which adaptation would differ when subjects interacted with multidimensional static and dynamic targets. A wooden dowel occupying the path followed by the falling balls and subtending a horizontal arc similar to that of the balls was used as a target in both training and testing conditions.
In training conditions, the dowel extended downward far enough for the subject's fingers to come into contact with it when he/she pointed at it. During test trials, the dowel did not extend as far down, and the subjects could point at, but not feel, the location of the dowel.

Method
Subjects
Forty-four undergraduates participated in partial fulfillment of
an introductory psychology course's requirements. None of the sub-
jects had participated in Experiment 1 . The data from 4 subjects
were discarded due to their failure to keep their eyes closed while
the target dowel was moved between testing positions; these sub-
jects were removed because viewing the movement of the dowel
might provide dynamic information.

\section{Apparatus}

During the training phase, a wooden dowel $(2.7 \mathrm{~cm}$ in diameter and $77.75 \mathrm{~cm}$ in length) was affixed to the drop bar, at the same position used for training in Experiment $1(+8.2 \mathrm{~cm})$. The dowel ended $6.5 \mathrm{~cm}$ above the upper edge of the hand opening. In order to stabilize the dowel during training, it was secured to a removable board $6 \mathrm{~cm}$ above the end of the dowel. When the subject's hand swung out through the hand opening, the hand did not contact the dowel; however, after the hand's horizontal motion was halted, it was possible to contact the bottom of the dowel by extending the index finger upward. During the testing phase, a shorter dowel $(66.5 \mathrm{~cm}$ in length) was used. This provided the same visual stimulation as the training dowel, but it prevented haptic feedback.

\section{Procedure}

Procedures were identical in all respects to those of the minimumprism, minimum-standard, maximum-prism, and maximumstandard groups of Experiment 1, with the following changes: Instructions were changed to reflect the change from rubber balls to wooden dowel as target. The experimenter paced responding by calling "knee" (the subject placed his/her right hand on the left knee), "point" (right hand moved to a point the subject judged to be under the dowel), "contact" (index finger was extended upward and either touched the dowel or did not). The subject was instructed to say "miss" when the index finger did not touch the dowel; the subject was instructed not to move the hand around to find the target after extending the index finger upward.

During the testing phase, the subject was instructed to place the index finger directly under the dowel as he/she had in the training phase, but, because the dowel was shorter, he/she need not extend the finger upward. After the subject had responded, he/she was told to close his/her eyes until the experimenter asked him/her to open the eyes and to point. During this interval the dowel was moved to 1 of the 13 test positions used in Experiment 1. The 13 positions were tested in random order. This was repeated three times, for a total of 39 test trials.

\section{Results and Discussion}

Adaptation to static stimuli appears qualitatively different from adaptation to dynamic stimuli. In Experiment 2 , the patterns of generalization for static stimuli were different from the patterns seen in Experiment 1. The effects of prism experience with static stimuli was assessed by examining the differences in test trial performance between the groups trained with prism goggles and those trained with standard goggles (see Figure 6). As in Experiment 1, difference scores for both occluder 


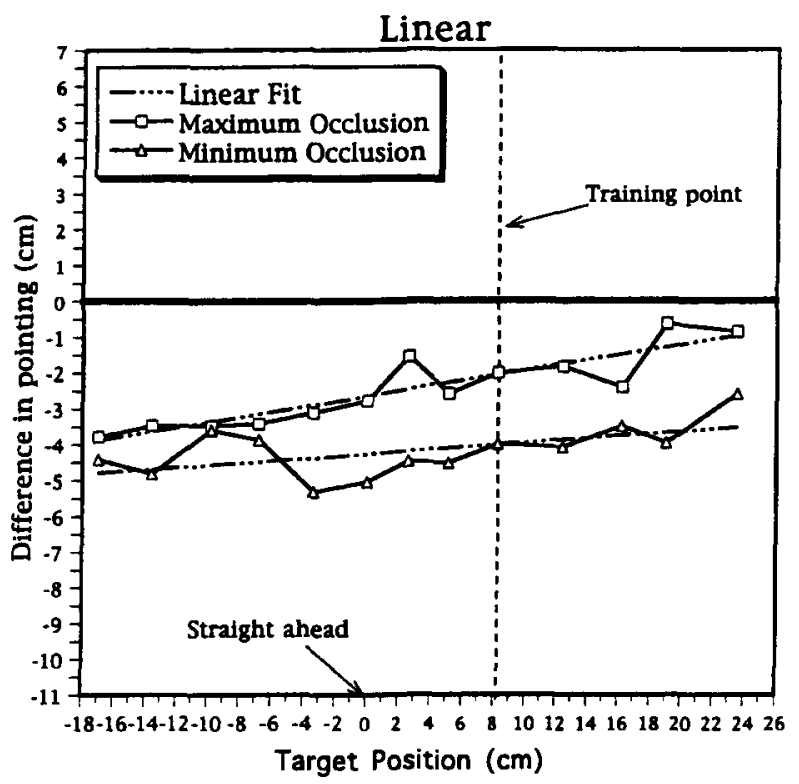

Figure 6. Pointing differences between the standard and prism groups in Experiment 2. The mean pointing difference between the standard and prism groups is plotted as a function of dowel position for the two occluder heights. Also indicated are the bestfitting linear functions for the pointing differences between the prism and standard groups.

heights were calculated by subtracting the standard group means from the prism group means at each of the 13 positions. Two aspects of Figure 6 are important. First, there appears to be no clear spatial gradient in the generalization of adaptation from trained to untrained locations with either the minimum occlusion groups or the maximum occlusion groups. That is, the pattern of responses across the 13 test positions is a flat function (see Figure 6). Second, for the minimum occlusion groups, the adaptation aftereffects from the static environment appear weaker than those seen following interactions with the dynamic environments. For the maximum occlusion groups, on the other hand, the differences in pointing to static stimuli appear remarkably similar to those obtained with the maximum occlusion groups with dynamic stimuli.

An examination of within-group variability (the standard deviations of pointing errors) revealed similar patterns of variability as those found in Experiment 1. That is, variability appeared fairly uniform across all target positions.

The results of Experiments 1 and 2 reveal basic differences in how adaptation occurs with respect to dynamic and to static events. Adaptation differed in both the extent of adaptation at the trained location and the nature of generalization of adaptation to untrained locations. Previous research has noted differences in the behaviors that adapt in dynamic and static arrays. Wallace (1975) found that adaptation of two different responses differed for moving and static training stimuli. The two responses he examined were visually locating (the experimenter moved a target, and subjects stated when it appeared to be straight ahead) and pointing (with their arm hidden from view, subjects pointed to what they thought was the straight-ahead position). He found greater adaptation for visually locating after training with moving objects and greater adaptation for pointing after training with stationary objects. Wallace did not address the issue of differences between trained and untrained locations. An important question for future research is whether or not these two behaviors will show similar patterns of generalization in the dynamic and static domains.

The subjects' pointing errors (the difference between the actual location of the ball and the location where the subject pointed) were submitted to a four-way ANOVA, with type of goggles and occluder height as betweensubjects factors, and test series and target position as within-subjects factors. As in Experiment 1, the significant main effect of goggles $[F(1,36)=8.18, p<.007]$ indicates that adaptation occurred. As was also found in Experiment 1 , the main effects of test series and position were significant $[F(2,72)=9.91, p<.0002$, and $F(12,432)=$ $7.29, p<.0001$, respectively]. While the main effect of occluder height was not significant $[F(1,36)=1.26, p>$ $.26]$, the same pattern found in Experiment 1 was present in Experiment 2: Adaptation tended to be stronger for the minimum occlusion group. None of the interactions were significant.

The main effect of position was further explored with trend analyses. These analyses were conducted in an identical manner to those conducted for Experiment 1. More specifically, two-way ANOVAs were performed on the differences between the individual scores in the prism group and the mean score for each of the 13 target locations for the standard group. For the minimum occlusion group, neither the main effect of test series nor the main effect of position were significant $(F \mathbf{s}<1)$ : The subjects' performance did not vary as a function of target location or as a function of repeated test trials. Trend analyses were not performed for the minimum occlusion group, since there was no effect of position.

For the maximum occlusion group, the main effect of position was significant $[F(12,108)=3.73, p<.0001]$, and the main effect of test series was not significant $[F(2,18)=3.3, p<.06]$. The interaction was not significant. Only the linear trend was significant [for the linear trend, $F(1,108)=36.8, p<.0001$; for the five higher orders, $F_{\mathrm{s}}(1,108)<1.97$, ps $\left.<.17\right]$. Thus, the variation in response across position was completely linear in nature.

To compare the patterns of adaptation found in Experiments 1 and 2, the subjects' pointing errors were subjected to a four-way ANOVA, with stimulus (falling ball and wooden dowel) and occluder height (maximum and minimum) as between-subjects factors, and test series (3 levels) and target position (13 levels) as within-subjects factors. As expected on the basis of a visual examination of Figures 4 and 6 , there was a stimulus $\times$ position interaction $[F(12,432)=4.22, p<.0001]$, indicating that the 
pattern of errors across position was different for dynamic and static stimuli, an occluder height $\times$ position interaction $[F(12,432)=4.56, p<.0001]$, indicating that the pattern of errors across position was different for different occluder heights, and a nonsignificant threeway stimulus $\times$ occluder height $\times$ position interaction $[F(12,432)=1.74, p<.06]$. These interactions confirm our observation that the pattern of adaptation found across the positions depends on the nature of stimulus (dynamic or static) and the occluder height. To explore the effect of dynamic and static stimuli under different conditions of occlusion, separate ANOVAs were run for the two levels of occluder height. Under maximum occlusion, there was no difference in generalization across position between static and dynamic stimuli with maximal occlusion-the stimulus $\times$ position interaction was not significant $(F<1)$. In contrast, there was a clear difference in generalization across position with minimal occlusion [stimulus $\times$ position interaction, $F(12,216)=$ $4.22, p<.0001]$. Simple effects analysis indicated that the degree of adaptation found in dynamic stimuli was significantly greater for 3 of the locations (Positions 5, 6, and 7$)[F(1,35) \geq 5.16, p<.05]$.

In summary, adaptation to the static arrays in Experiment 2 yielded results similar to those reported by Bedford (1989), in that there was a strong linear component in both minimum and maximum occlusion conditions. The linear function with static stimuli suggests that the dynamic nature of the targets, rather than some other procedural aspect of Experiment 1, was responsible for the graded functions. Specifically, a comparison of results from Experiments 1 and 2 indicates that adaptation to dynamic environments differs from adaptation to static environments, in that, with dynamic targets that are minimally occluded, the extent of adaptation at the training location is greater than that seen with static stimuli, and generalization of adaptation decreases as the distance increases from the training location.

\section{EXPERIMENT 3 Training All Positions}

Experiment 3 was designed to rule out the possibility that our results with dynamic stimuli arose from some artifact of the training or testing procedures. To find out whether these curves were specific to the training used in Experiment 1, the subjects in Experiment 3 were trained at all of the 13 test positions employed in Experiment 1. Since the greatest effects in Experiments 1 and 2 were observed in the minimum occlusion condition, only this condition was employed in Experiment 3.

\section{Method}

\section{Subjects}

Twenty-three subjects were recruited from undergraduates responding to posted advertisements for experimental subjects and were paid for their participation in this experiment. None of the

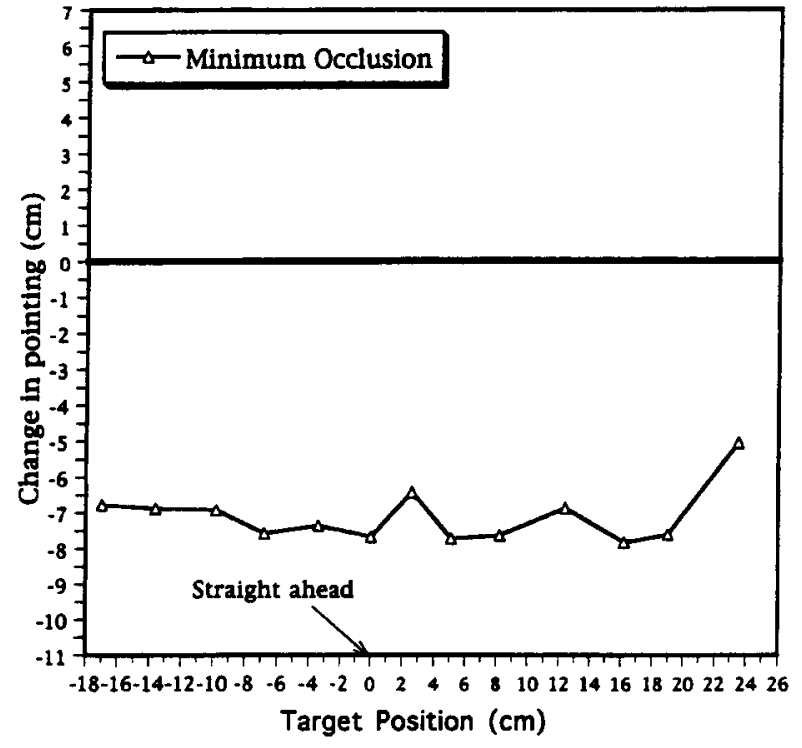

Figure 7. Pointing differences between the prism and standard groups in Experiment 3 following training at all locations using the minimum occluder screen. The mean pointing location differences are plotted as a function of ball-dropping position.

subjects had participated in Experiment 1 or Experiment 2. The data from 3 of the subjects were discarded due to failure to meet the training criteria for at least 1 of the 13 positions.

\section{Procedure}

Procedures were identical in all respects to those of the minimum prism and minimum-staridard groups from Experiment 1, with the following exceptions: (1) Training was provided at all 13 locations. (2) The completion criterion for each position was set at a minimum of 15 trials with at least 3 of the last 6 trials correct (i.e., ball touches the index finger). (3) Each position was trained to criterion prior to moving to the next, with order of positions randomized for each subject.

\section{Results and Discussion}

The differences between prism and standard groups trained at all locations are shown in Figure 7 . A consistent difference in pointing is seen across all positions. This function is clearly linear and obviously different from the one found in Experiment 1. The adaptation at the rightmost position was somewhat less than the others, suggesting there might have been some mechanical constraints on motion in the apparatus such that movements to the extreme right were difficult. However, no statistically significant differences were found across positions. The main effect of positions in a two-way ANOVA performed on the difference scores did not approach significance $[F(12,108)=1.297, p>.2]$. The results of Experiment 3 clearly indicate that the curvilinear functions seen in the data from Experiment 1 were a function of training occurring at a single point and were not attributable to a mechanical constraint or other artifact peculiar to the apparatus or procedure. 


\section{EXPERIMENT 4 Training Without Haptic Feedback}

Wallach (1968) identified two general classes of explanations for how humans learn a new relationship between two sources of information for the same physical quality (e.g., where in space an object is seen and felt). Wallach (like most other researchers) focused his attention on explanations that involved learning a new relationship between the two sources of information based on some form of feedback. A second class of explanations was suggested by some of Gibson's early research. Gibson (1933) found that prolonged viewing of curved lines resulted in a reduction of apparent curvature. He also found a negative aftereffect immediately following such exposure, with straight lines appearing curved (Gibson, 1937). Similarly, Hess and Doshi (1995) found that, after visual exposure to a three-element display with the middle element offset, subjects will report the middle element as offset when they later see the same three elements in alignment. The adaptation seen in such studies may have a normative basis. For example, lines tend not to all be curved; so when confronted with a world where the population of lines all tend to curve in one direction, perception changes so that the lines no longer have any systematic bias. In such a case, adaptation occurs in the absence of motor responses to the stimuli and subsequent feedback.

Prism adaptation to static displays has typically involved visual feedback; however, a few studies have employed haptic feedback (e.g., Howard et al., 1965; Lackner, 1974; Welch, 1978). Howard et al. (1965) demonstrated adaptation when subjects viewed the approach of a rod that was displaced by a prism and then felt it touch their lips. When Lackner (1974) trained subjects wearing prisms to point below a table at vertical rods that extended above and below the table top, he found adaptation with subjects who could not see their hands but could feel the rods when they pointed directly at them. In contrast, Welch (1978) replicated Lackner's procedures but added conditions that allowed comparisons of adaptation with visual feedback and adaptation with haptic feedback. He found haptic information to be relatively ineffectual for producing adaptation and concluded that "tactual feedback is a relatively poor source of prism adaptation" (Welch, 1978, p. 35).

In Experiment 1, adaptation was found when only haptic feedback was available. Before concluding that haptic feedback had a robust effect, we must demonstrate that the feedback was necessary. In Experiment 4, we examined the extent to which adaptation would occur in our preparation given exposure to the same experimental procedures as in Experiment 1, but without haptic feedback for correct pointing.

\section{Method}

\section{Subjects}

Twenty undergraduates participated in partial fulfillment of an introductory psychology course's requirements. None had participated in the previous experiments.

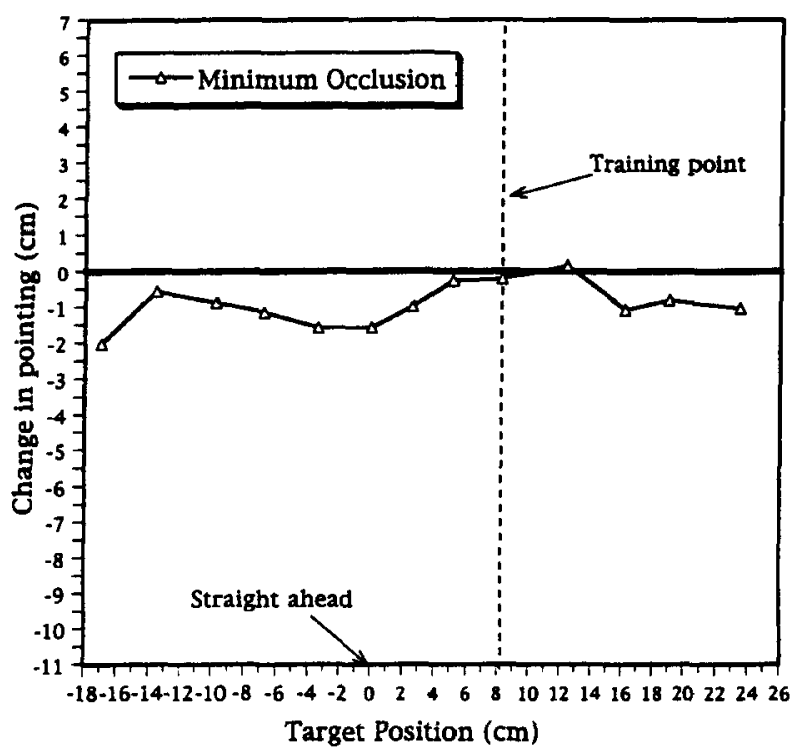

Figure 8. Mean pointing differences between the prism and standard groups following training without feedback in Experiment 4.

\section{Procedure}

Procedures were identical in all respects to those of the minimum-prism and minimum-standard groups of Experiment 1, with the following exceptions: (1) The cushioned tray remained in place throughout training and testing so the subjects' hands never contacted the ball. (2) All subjects were given 70 training trials (no accuracy criterion was employed).

\section{Results and Discussion}

As can be seen in Figure 8, in the absence of feedback, no systematic differences were found between groups trained with prisms and those trained without. Clearly, the haptic consequences of correct and incorrect intercepting movements were critical for adaptation and functioned as an extremely effective mode of feedback in producing adaptation to prism-displaced events.

\section{EXPERIMENT 5 Within-Subjects Replication}

Bedford $(1989,1993 a)$ and many other researchers (see Harris, 1965, and Welch, 1978, for reviews) utilized within-subjects designs with comparisons of pretraining and posttraining tests. One might speculate that the differences between Bedford's and our results are in some way related to the between-groups comparisons employed in our Experiments 1-4. In Experiment 5, we replicated the condition with the largest effect from Experiment 1 using a within-subjects design and examined the change in pointing for each subject following training with prism goggles in the minimum occlusion condition.

\section{Method}

\section{Subjects}

Twelve undergraduates participated in partial fulfillment of an introductory psychology course's requirements. None had partici- 


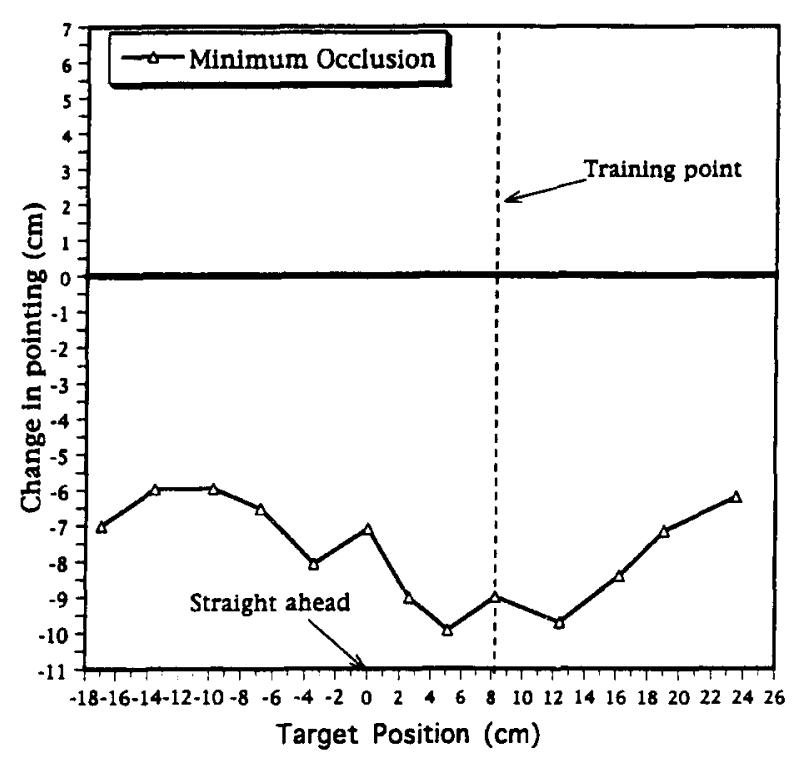

Figure 9. Mean within-subjects change in pointing following training at a single location in Experiment 5. Differences between pretraining and posttraining tests are plotted as a function of balldropping position. All subjects were trained with prism goggles using the minimum occluder screen.

pated in any of the previous experiments. The data from 2 subjects were discarded: one due to failure to meet the training criteria, and another because she was able to report, during postsession questioning, a difference between the goggles used during training and those used during testing.

\section{Procedure}

Procedures were identical in all respects to those of the minimumprism group of Experiment 1, with the following changes: (1) A pretest was administered prior to training in which all subjects wore the standard goggles and were given one trial at each of the 13 positions (order randomized). As in posttraining test trials, the cushioned tray was in place during these trials to prevent haptic feedback from contact with the balls. (2) The order of instructions was rearranged and given in two parts, such that the instructions used in Experiment 1 for test trials were delivered prior to the pretest and again prior to the posttest, and the training instructions were given between pretest and training.

\section{Results and Discussion}

The data were analyzed by computing the difference between each subject's pretest score and their posttest scores at each position. Figure 9 depicts the mean difference scores. A generalization gradient centered around the training position can be seen here; its appearance is very similar to the difference scores calculated between groups for the minimum occlusion condition in Experiment 1 . The fit for a Gaussian function was slightly lower than that found in Experiment $1\left(r^{2}=.83\right)$. However, the linear fit $\left(r^{2}=.12\right)$ was also reduced. There are some minor differences between these results and those of the minimum occlusion group of Experiment 1; however, it is clear that the adaptation was graded as a function of the distance from training position.

\section{GENERAL DISCUSSION}

The present findings demonstrate the occurrence of adaptation to visual displacement of dynamic targets under conditions in which only haptic feedback is available. The extent of adaptation increased as the visible path of the falling object increased. Additionally, the extent of adaptation varied as a function of distance from the training location, with the greatest adaptation found at and around the training point. The variation in adaptation was well fit with a Gaussian curve. In contrast, adaptation to formally similar static displays showed a uniform linear generalization across positions. Thus, the generalization of adaptation differed with respect to dynamic and static arrays. The results of Experiments 3, 4, and 5 indicate that the pattern of adaptation found in Experiment 1 reflects a gradated change in adaptation as the distance between the training and testing events increased; the results were not due to aspects of the experimental preparation, such as mechanical constraints on motion, changes in behavior that occur in the absence of feedback, or within-group versus between-groups comparisons.

The curvilinear functions found in the present series of experiments appear to depart markedly from earlier observations (Bedford, 1989, 1993a) of how training at a single location will generalize to responding to targets at untrained locations. Bedford only found nonlinear generalization gradients when she used a procedure she describes as cognitive (Bedford, 1993a, Experiments 3 and 4). In those procedures, subjects were trained at a number of positions, but the task was presented as a problem solving task. Subjects were told that they were to learn where to point when lights came on at various positions, thus emphasizing the possibility of a discrepancy between where a position was located and where they were to point (this was reinforced by the fact that the LED on the tip of the finger would not be aligned with the target when it lit up). In those experiments, Bedford found that when the subjects knew ihat nonveridical relations (between visual and proprioceptive space) were possible, a steep generalization gradient at or near the nonveridical position (or, in her Experiment 4, the only veridical position) was evidenced. Knowledge of an altered relationship between visual and proprioceptive space cannot explain the present curvilinear functions, since we have systematically excluded the only 3 subjects that could describe a difference between goggles with and without prism lenses.

It is possible for a generalization gradient, such as the ones found here, to be the product of different response patterns in individual subjects. If, for example, each subject showed maximum adaptation at the point of training and a linear decline in adaptation to one side or the other, but not both sides, then the combined responses of the two groups of subjects would show a gradual decline in adaptation to the left and right of the training location. However, the responses we observed in Experiments 1 and 5 were not of this nature. Such a pattern of responding would result in a local minima in variability at the 
point of training. As is evident in Figure 3 (which shows the standard deviation of subject's pointing across the 13 test locations), in Experiment 1, there was no sign that the variability in responding was substantially higher adjacent to the training location. Furthermore, the variability in the prism and standard groups did not differ appreciably. The patterns of variability seen in all of these experiments suggest that the curvilinear response represents gradations in the degree of adaptation seen across space, and not a mixture of two response patterns.

Another difference between our dynamic targets and the static targets in Bedford's studies, as well as in our Experiment 2, is the presence or absence of temporal gaps between antecedent and consequent stimulation. It might be tempting to suppose that the relevant difference, accounting for linear versus nonlinear functions, was the temporal separation between seeing the target and subsequent feedback, with linear functions seen when feedback was simultaneous. However, an examination of the curves generated by the three occluder heights in Experiment 1 indicates an opposite pattern of results: As the temporal distance between seeing and feeling the ball decreased, the curvilinear generalization gradient increased.

Interestingly, the magnitude of adaptation did appear to be a function of the spatiotemporal separation between seeing and feeling the ball. When the separation was small $(23.5 \mathrm{~cm}$ and $64 \mathrm{msec})$, strong adaptation was seen; as the separation increased, the level of adaptation decreased. When the separation was largest $(70.5 \mathrm{~cm}$ and $256 \mathrm{msec}$ ), in the maximum occlusion condition, the level of adaptation around the training position was approximately one third that seen in the minimum occlusion condition. Temporal and spatial gaps are known to affect both perception and learning. For example, both temporal and spatial gaps affect the perception of identity and motion of discontinuously presented moving objects (Michotte, Thines, \& Crabbe, 1964; Neff, 1930). Temporal distances also affect how the development of Pavlovian (e.g., Kimble, 1947; Macintosh, 1974) and operant (e.g., Sidman, 1969; Sizemore \& Lattal, 1977) relations proceed, with larger delays resulting in weaker effects. Adaptation in the present context requires haptic feedback, as demonstrated by Experiment 4 , and it is possible that the separations influenced the effectiveness of the haptic consequences. Although we did not attempt, here, to determine the relative roles played by temporal and spatial gap size, we believe that it is an important issue for future research.

In Experiment 2, we examined another possible cause of the differences noted between our findings in Experiment 1 and Bedford's: the higher dimensionality of our stimuli. However, when subjects interacted with multidimensional static targets that provided static information that was formally similar to the information provided by the dynamic targets, we did not find curvilinear functions. Instead, we obtained linear patterns of results that were similar to those obtained by Bedford.

What, then, might account for the decrements in generalization of adaptation to dynamic events? There appears to be at least three possible reasons that dynamic stimuli produce decreased generalization and increased specificity relative to static stimuli. First, dynamic and static environments may offer differing types of optical information. Second, action with respect to the two environments often requires different characteristics. Third, development of successful interactions with static and moving objects may be fundamentally different enough to result in different behavior with respect to any new instance of one or the other. These are clearly not mutually exclusive, and patterns of generalization might be influenced by any of these three factors. We briefly consider each of these possibilities below.

The visual information present in the dynamic stimuli might have contributed to a greater degree of discrimination than Bedford found. Researchers in the area of event perception have noted that dynamic displays are sources of multidimensional information not available in static displays. Peper et al. (1994) proposed that changes in the optical array (e.g., optical image size, optical specification of passing distance, and angle of approach) can specify the spatiotemporal locations that a moving object will occupy. In order to behave effectively in everyday interactions with moving objects, one must be sensitive to the speed, changes in speed, trajectory, and changes in trajectory. Thus, apparent path, alone, does not sufficiently specify an effective point of interception for a moving object. Dynamic events, such as those in Experiment 1 , are more complex than their corresponding static arrays (as in Experiment 2). This added complexity may effectively make spatially proximal dynamic events more dissimilar than corresponding spatially proximal static paths. Thus, the occurrence of adaptation would be restricted to a more local region.

The second factor that may have contributed to the differing findings concerns the functional differences between the pointing responses in Bedford's studies, the touching responses with our static targets, and the intercepting responses with dynamic targets. To point and touch effectively requires an accurate correspondence between the current target position and the angular direction of the pointing hand (touching also requires accurate radial distance). In contrast, effective interception requires an accurate correspondence between the location of where the target will be at some point in the future, the angular direction of the hand, and the radial distance from the body. That is, the functional success of a pointing or reaching response does not depend on when it occurs, but the effectiveness of an intercepting response depends on its occurrence within a set temporal window. So, although the topography of these responses might look very similar for a constrained catching situation such 
as the one employed here, intercepting is necessarily controlled by a more complex set of relationships than pointing or reaching.

The third possibility arises from the first two and concerns the differences in how we learn to deal effectively with moving and stationary objects. To the extent that our histories with respect to the two classes of interactions differ, our subsequent behavior may differ. Indeed, how new learning, and its generalization, proceeds may differ. That is, dynamic and stationary objects become members of two separate classes of events, with differing repertoires brought to bear on each. Any parent can attest to the extraordinary difficulty of teaching a toddler to catch a ball. A child who has become quite adroit at reaching for and navigating among stationary objects is still quite unprepared to intercept a thrown ball. A new set of skills must develop to effectively interact with thrown balls. What may eventually be learned is that moving things are to be treated differently than stationary ones, including how to adapt to them when there are changes. The reasons for treating them differently lie with the prior two reasons - the added complexity that requires different behavior with respect to changing arrays. For instance, moving objects tend not to precisely follow the same path, and, because they are less predictable than stationary ones, they must be dealt with somewhat more idiosyncratically. Additionally, animals are prevalent dynamic objects that follow complex paths that are unpredictable at another level. Thus, even though a simple linear transformation of arm movements would have been as effective for the current dynamic targets as it was for the wooden dowels, the subjects' prior experiences with moving objects, in general, might have limited the adaptation to the area of the recent training interactions.

At a very basic level, the discrepancies between the present results and Bedford's results concern the conditions under which generalization occurs locally or more globally. There is a substantial body of research and literature addressing that specific topic under the rubrics of stimulus control, discrimination, and generalization (for reviews, see Dinsmore, 1995a, 1995b; Mostofsky, 1965; Nevin, 1973; Terrace, 1966). ${ }^{3}$ At a theory-free level, discrimination is behaving differently in different situations, whereas generalization is behaving similarly in different situations (Hineline, 1992). Although a review and conceptual integration of that body of work with the area of perceptual adaptation is beyond the scope of this paper, we would like to suggest that theoretical interpretations of generalization of adaptation would benefit from their consideration. The three factors (i.e., stimulus complexity, behavioral function, and past experience) proposed as contributing to these observed differences have each received attention within the stimulus control domain.

As an example of the applicability of these areas, generalization gradients similar to the present gradients have been found in many discrimination experiments. For example, when Honig, Boneau, Burstein, and Pennypacker (1963) trained pigeons to peck a key with a single line orientation and then tested their rate of pecking to keys with varying line tilts, they found smooth generalization gradients in rate of pecking, with the greatest rates of pecking at the trained orientation. Similar gradients have been found along numerous dimensions (e.g., wavelength of light, Hanson, 1959; sound frequency, Jenkins \& Harrison, 1960; and temporal distance, Sidman, 1969). Moreover, Fink and Patton (1953) found broad generalization gradients for unidimensional stimulus variation, whereas much narrower gradients were evident with variation along three dimensions. As the complexity of change increases, generalization becomes less global.

Observations of generalization decrements led some early learning theorists (e.g., Estes, 1950; Spence, 1936) and their modern connectionist counterparts (e.g., Rumelhart \& McClelland, 1986) to propose models that are based on learning elementary, pairwise relationships-either between pairs of stimuli or between a stimulus and response. However, other learning theorists (e.g., Catania, 1973, 1992; Sidman, 1986; Skinner, 1935, 1965, 1969) explicitly reject pairwise associations and have found it more useful to construe these observations as indicative of the development of relations between classes of behavior and multidimensional aspects of the environment. In a similar manner, Bedford rejected an account of prism adaptation based on the developing of separate connections between visual and proprioceptive stimuli, and she suggested that relationships between dimensions, rather than pointwise associations, were learned. ${ }^{4}$ She also proposed that there is a linear constraint on the adaptation process, "such that what is changed through experience is the value of a slope and of an intercept parameter" (Bedford, 1989, p. 245). She argued that her findings were indicative of how organisms adapt to changes that result in visual-proprioceptive discordance and, more generally, are characteristic of perceptual learning. Although we would also reject an interpretation based on learning individual pairwise connections, the present experiments and analyses appear to indicate limits to the generality of her linear constraints model. The discrepancy in findings between static and dynamic procedures presents an interesting empirical challenge: determination of the critical differences between interactions with static and dynamic events that yield such discrepancies. The question of whether a general law of perceptual learning can be formulated that can encompass both sets of findings may have to await identification of the relevant differences between the two procedures.

Finally, the results of the present experiments clearly indicate that haptic feedback can be effective in producing adaptation to visual displacement. As noted above, there has been a lack of consensus between researchers studying haptic versus visual feedback, with some finding haptic feedback effective (e.g., Howard et al., 1965; Lackner, 1974, 1977) and others finding it relatively ineffective (e.g., Healy, Symmes, \& Ommaya, 1973; Welch, 1978). Although we did not specifically compare visual feedback with haptic feedback, we did find that, with dy- 
namic targets in our minimum occlusion condition, adaptation at the training locations was very high. Haptic consequences, under these conditions, appear to be extremely effective in facilitating adaptation, and no adaptation appears to occur in their absence (Experiment 4).

The differences in adaptation to dynamic and static displays clearly merit further consideration. Future investigation of adaptation to dynamic events should disentangle the relative roles of temporal and spatial distance between visual and haptic experience. Integration of prism adaptation and the theoretical and empirical work on stimulus generalization may prove useful in these investigations. The present study also indicates the importance of continued explorations of the conditions under which various modes of feedback function in adaptation to visual displacement.

\section{REFERENCES}

BaILY, J. S. (1972). Adaptation to prisms: Do proprioceptive changes mediate adapted behavior with ballistic arm movements? Quarterly Journal of Experimental Psychology, 24, 8-20.

BeCKetT, P. A., \& MElamed, L. E. (1980). Further evidence dissociating decay and readaptation in prism adaptation. Bulletin of the Psychonomic Society, 16, 73-75.

BEDFORD, F. L. (1989). Constraints on learning new mappings between perceptual dimensions. Journal of Experimental Psychology: Human Perception \& Performance, 15, 232-248.

BEDFORD, F. L. (1993a). Perceptual and cognitive spatial learning. Journal of Experimental Psychology: Human Perception \& Performance, 19, 517-530.

Bedford, F. L. (1993b). Perceptual learning. Psychology of Learning \& Motivation, 30, 1-60.

CATANIA, A. C. (1973). The concept of the operant in the analysis of behavior. Behaviorism, 1, 103-116.

Catania, A. C. (1992). Learning (3rd ed.). Englewood Cliffs, NJ: Prentice-Hall.

CoHen, H. B. (1966). Some critical factors in prism-adaptation. American Journal of Psychology, 79, 285-290.

Dinsmore, J. A. (1995a). Stimulus control: Part I. The Behavior Analyst, 18, 51-68.

DiNSMORE, J. A. (1995b). Stimulus control: Part II. The Behavior Analyst, 18, 253-269.

Estes, W. K. (1950). Toward a statistical theory of learning. Psychological Review, 57, 94-107.

Fink, J. B., \& PATton, R. M. (1953). Decrement of a learned drinking response accompanying changes in several stimulus characteristics. Journal of Comparative \& Physiological Psychology, 46, 23-27.

GiBson, J. J. (1933). Adaptation, after-effect and contrast in the perception of curved lines. Journal of Experimental Psychology, 16, 1-31.

GiBson, J. J. (1937). Adaptation with negative after-effect. Psychological Review, 44, 222-244.

Goldstein, S. R., \& HALL, D. (1990). Variable ratio control of the spitting response in the archer fish (Toxotes jaculator). Journal of Comparative Psychology, 104, 373-376.

HANSON, H. M. (1959). Effects of discrimination training on stimulus generalization. Journal of Experimental Psychology, 58, 321-334.

HARRIS, C. S. (1965). Perceptual adaptation to inverted, reversed, and displaced vision. Psychological Review, 72, 419-444.

HARRIS, C. S. (1980). Insight or out of sight? Two examples of perceptual plasticity in the human adult. In C. S. Harris (Ed.), Visual coding and adaptability (pp. 95-149). Hillsdale, NJ: Erlbaum.

Healy, M. H., Symmes, D., \& OMmaYA, A. K. (1973). Visual discordance cues induce prism adaptation in normal monkeys. Perceptual \& Motor Skills, 37, 683-693.

HELD, R. (1961). Exposure history as a factor in maintaining stability of perception and coordination. Journal of Nervous \& Mental Disease, 132, 26-32.

Held, R., \& Hein, A. (1958). Adaptation to disarranged hand-eye coordination contingent upon re-afferent stimulation. Perceptual \& Motor Skills, 8, 87-90.

Hess, R. F., \& Doshi, S. (1995). Adaptation to spatial offsets, Perception, 24, 1407-1426.

Hineline, P. N. (1992). A self-interpretive behavior analysis. American Psychologist, 47, 1274-1286.

Honig, W. K., Boneau, C. A., Burstein, K. R., \& Pennypacker, H. S. (1963). Positive and negative generalization gradients obtained after equivalent training conditions. Journal of Comparative \& Physiological Psychology, 56, 111-116.

Howard, I., CRaske, B., \& Templeton, W. (1965). Visuomotor adaptation to discordant exafferent stimulation. Journal of Experimental Psychology, 70, 189-191.

Jenkins, H. M., \& HaRRISON, R. H. (1960). Effect of discrimination training on auditory generalization. Journal of Experimental Psychology, 59, 246-253.

KaISER, M. K., \& PHATAK, A. V. (1993). Things that go bump in the light: On the optical specification of contact severity. Journal of Experimental Psychology: Human Perception \& Performance, 19, 194-202.

Kaiser, M. K., \& Proffitt, D. R. (1984). The development of sensitivity to causally relevant dynamic information. Child Development, 55, 1614-1624.

Kim, N. G., Turvey, M. T., \& CAREllo, C. (1993). Optical information about the severity of upcoming contacts. Journal of Experimental Psychology: Human Perception \& Performance, 19, 179-193.

Kimble, G. A. (1947). Conditioning as a function of the time between conditioned and unconditioned stimuli. Journal of Experimental Psychology, 37, 1-15.

LACKNER, J. R. (1974). Adaptation to displaced vision: Role of proprioception. Perceptual \& Motor Skills, 38, 1251-1256.

LACKNER, J. R. (1977). Adaptation to visual and proprioceptive rearrangement: Origin of the differential effectiveness of active and passive movements. Perception \& Psychophysics, 21, 55-59.

LEE, D. N., \& REDDISH, P. E. (1981). Plummeting gannets: A paradigm of ecological optics. Nature, 293, 293-294.

MaCintosh, N. J. (1974). The psychology of animal learning. New York: Academic Press.

MCBeATH, M. K. (1990). The rising fast-ball: Baseball's impossible pitch. Perception, 19, 545-552.

MCBeath, M. K., Shaffer, D. M., \& Kaiser, M. K. (1995). How baseball outfielders determine where to run to catch fly balls. Science, $\mathbf{2 6 8}$, 569-573.

Melamed, L. E., Moore, L. A., \& BeCKetT, P. A. (1979). Readaptation and decay after prism viewing: An exploration of task variables from the viewpoint of the information discordance hypothesis. Perception \& Psychophysics, 26, 215-220.

Michaels, C. F., \& Oudejans, R. R. D. (1992). The optics and actions of catching fly balls: Zeroing out optical acceleration. Ecological Psychology, 4, 199-122.

Michotte, A., Thines, G., \& Crabbe, G. (1964). Les complements amodaux des structures perceptives [Amodal completion of perceptual structures] (Studia Psycologica). Louvain: Publications Universitaires de Louvain.

Mikaelian, H. H. (1970). Adaptation to rearranged eye-foot coordination. Perception \& Psychophysics, 8, 222-224.

MosTOFSKY, D. I. (ED.) (1965). Stimulus generalization. Stanford, CA: Stanford University Press.

Moulden, B. (1971). Adaptation to displaced vision: Reafference is a special case of the cue-discrepancy hypothesis. Quarterly Journal of Experimental Psychology, 23, 113-117.

NEFF, W. S. (1930). A critical investigation of the visual apprehension of motion. American Journal of Psychology, 48, 1-42.

Nevin, J. A. (1973). Stimulus control. In J. A. Nevin \& G. S. Reynolds (Eds.), The study of behavior: Learning, motivation, emotion, and instinct (pp. 115-152). Glenview, IL: Scott, Foresman.

Peper, C. E., Bootsma, R. J., Mestre, D. R., \& Bakker, F. C. (1994) Catching balls: How to get the hand to the right place at the right 
time. Journal of Experimental Psychology: Human Perception \& Performance, 20, 591-612.

Redding, G. M., \& Wallace, B. (1995, November). Prism adaptation during ball throwing. Paper presented at the annual meeting of the Psychonomic Society, Los Angeles.

RedDing, G. M., \& Wallace, B. (1997). Adaptive spatial alignment. Mahwah, NJ: Erlbaum.

Rumelhart, D. E., \& MCClelland, J. L. (Eds.) (1986). Parallel distributed processing: Explorations in the microstructure of cognition. Cambridge, MA: MIT Press.

SHEPARD, R. N. (1984). Ecological constraints on internal representation: Resonant kinematics of perceiving, imagining, thinking, and dreaming. Psychological Review, 91, 417-447.

SIDMAN, M. (1969). Generalization gradients and stimulus control in delayed matching-to-sample. Journal of the Experimental Analysis of Behavior, 12, 745-757.

SIDMAN, M. (1986). Functional analysis of emergent verbal classes. In T. Thompson \& M. D. Zeiler (Eds.), Analysis and integration of behavioral units (pp. 213-245). Hillsdale, NJ: Erlbaum.

Sizemore, O. J., \& LatTal, K. A. (1977). Dependency, temporal contiguity, and response-independent reinforcement. Journal of the Experimental Analysis of Behavior, 25, 119-125.

SKINNER, B. F. (1935). The generic nature of the concepts of stimultis and response. Journal of General Psychology, 12, 40-65.

SKINNER, B. F. (1938). The behavior of organisms. New York: AppletonCentury.

SKINNER, B. F. (1965). Stimulus generalization in an operant: A historical note. In D. I. Mostofsky (Ed.), Stimulus generalization (pp. 193209). Stanford, CA: Stanford University Press.

SkinNer, B. F. (1969). The role of the environment. In B. F. Skinner (Ed.), Contingencies of reinforcement: A theoretical analysis (pp. 3-28). New York: Appleton-Century-Crofts.

SPENCE, K. W. (1936). The nature of discrimination learning in animals. Psychological Review, 43, 427-449.

TERRACE, H. S. (1966). Stimulus control. In W. K. Honig (Ed.), Operant behavior: Areas of research and application (pp. 271-344). New York: Appleton-Century-Crofts.

TREsilian, J. R. (1991). Empirical and theoretical issues in the percep- tion of time to contact. Journal of Experimental Psychology: Human Perception \& Performance, 17, 865-876.

WALLACE, B. (1975). Prism adaptation to moving and stationary target exposures. Perception, 4, 341-347.

WALLACH, H. (1968). Informational discrepancy as a basis for perceptual adaptation. In S. Freedman (Ed.), The neurophysiology of spatially oriented behavior (pp. 235-258). Homewood, IL: Dorsey.

WELCH, R. B. (1971). Discriminative conditioning of prism adaptation. Perception \& Psychophysics, 10, 90-92.

WELCH, R. B. (1978). Perceptual modification: Adapting to altered sensory environments. New York: Academic Press.

\section{NOTES}

1. Adaptation is used here as a procedurally defined term; it refers to changes in behavior that follows interactions with a new relationship between where things are seen to be and where they are felt. We do not address the locus of learning-whether the change affects the visual or motor systems. For a discussion of how these two types of changes might be distinguished, as well as theoretical issues relevant to the locus of the change, see Redding and Wallace (1997).

2. Lackner (1974) used a similar procedure to compare the efficacy of haptic and visual feedback in prism adaptation.

3. The applicability of operant conditioning principles to prism adaptation phenomena has been suggested previously. For instance, Welch (1971) demonstrated that prism adaptation can be brought under discriminative control. Additionally, an emphasis on function over topography, as noted in the previous discussion, is also consistent with operant conditioning apprcaches (see Skinner, 1938, 1965).

4. Bedford rejected a pointwise learning model based on her failure to find generalization gradients. We would also reject a model based on the learning of separate connections between visual and proprioceptive stimuli-however, not for the same reasons. The presence of nonlinear generalization gradients presents no difficulties for a sufficiently elaborated behavioral or perceptual learning theory.

(Manuscript received October 28, 1996; revision accepted for publication December 26,1997 .) 